\title{
Food in the digital platform economy - making sense of a dynamic ecosystem
}

Area of research interest: Emerging challenges and opportunities

Study duration: 2021-03-01

Planned completion: 31 May 2021

Project status: Completed

Project code: FS430653

Conducted by: University of Cambridge

Date published: 9 February 2022

DOI: https://doi.org/10.46756/sci.fsa.jbr429

\section{Background}

The food services sector has been evolving rapidly over the past decade, accelerated by the Covid-19 pandemic. The traditional linear model of food producers selling through wholesalers to brick and mortar retailers, restaurants and hospitality venues is increasingly being displaced by complex interactive digital ecosystems of online food services providers. Consumers are increasingly able to access food directly at various stages along the traditional value chain via interaction with digital platforms and rapid home-delivery networks, realising greater convenience, more variety in food products and services from a dynamic start-up scene, and overall enhanced value. FSA needs to stay abreast of these changes and develop regulatory responses to ensure these innovations are aligned with the public good and do not compromise food safety and public health.

\section{Research approach}

This report is a synthesis of desk research based on a review of the academic and grey literature and assimilation of previous Food Standards Agency (FSA) reports.

Analysis and review were undertaken using standard rapid evidence review protocols, and qualitative analysis where necessary.

\section{Results}

This review identified five key trends in food buying and selling in the digital platform economy:

\section{Online third-party platforms for food ordering and delivery}

These are aggregating platforms that enable consumers to browse a range of vendor offerings, place orders online, and arrange on-demand delivery within a certain geographic radius. Some of these platforms are well established, with brands such as Deliveroo, Uber Eats, Just Eat, and others, being already global household names. They are expanding rapidly and extending their reach into other areas of the food sector, including groceries deliveries, and provision of numerous new virtual restaurants. These platforms are enabling traditional brick and mortar businesses to easily enter the online economy and are facilitating a wealth of new entrants including online-only brands and home kitchens to enter the food sector. 


\section{Online marketplaces connecting buyers and food vendors}

Online marketplaces provide a venue for vendors to promote their products and services and act as an intermediary between buyers and sellers to facilitate transactions. These can be food marketplaces focusing exclusively on offering food products and services, such as focussing on speciality foods. Food event marketplaces offering culinary and experiential events such as supper-clubs and food tastings. General marketplaces where food and beverages are just a subset of the product range, such as Amazon. Social/open marketplaces that operate within a social media platform and facilitate peer-to-peer connections and exchanges. Redistribution marketplaces that seek to redistribute surplus food from farmers, producers, retailers, restaurants and consumers to reduce food waste. These marketplaces are enabling a wide array of new entrants to participate in the food sector.

\section{Direct producer/wholesaler to consumer commerce}

The past five years has seen a steep rise in the number of food producers and wholesalers developing direct-to-consumer (usually online) sales channels. These innovations bypass traditional intermediaries, shorten supply-chains, and enable consumers to connect directly with producers such as local farms and specialty ingredient suppliers. Notable innovations in this space include farm drops (providing fresh produce direct from the farm), and a wide range of recipe boxes and meal boxes that are redefining the boundaries between traditional grocery shopping and ready-made meals and delivering an experiential aspect for consumers.

\section{Dark kitchens}

Dark kitchens, alternatively known as cloud kitchens or ghost kitchens, are large-scale restaurant style food preparation spaces that do not have a customer-facing store front and operate a business-to-business (B2B) model by making the space available/outsourced to restaurants and brands that require extra capacity. They are typically located in low-cost but central areas in the urban environment, to enable rapid servicing of their local markets. Facilities have minimal staffing levels, and are optimised for high through-put, with the ability to be reconfigured quickly and easily to react to emerging market trends. Dark kitchens may be owned by an existing brick and mortar establishment to augment capacity, or as is increasingly the case, are online-only operating as virtual brands, multi-brand kitchens, or providing third-party food services to other operators under franchise models. Several major players are entering this market in the UK offering outsourced dark kitchens much like co-working spaces, enabling food entrepreneurs to enter the market with minimal setup costs and risk.

\section{Rapid on-demand delivery solutions}

The industry is building quick-commerce, or q-commerce solutions, that aim to cut on-demand delivery times to 15 minutes or less to make home-delivery the preferred choice for consumer food and convenience needs. This is being achieved through a combination of locally positioned restaurants and stores, leveraging dark kitchens and dark stores (centrally located fulfilment warehouses), and local courier networks. Use of innovative transport solutions such as escooters, autonomous robots and aerial drones are already being tested or are in operation offering rapid and low-carbon deliveries. These developments are transforming the traditional food buying and selling infrastructure into a dynamic network ecosystem of digital transaction hubs that will be able to quickly respond to novel consumer demands and trends across a wide range of sectors of the economy.

\section{Longer-term trends}

Looking forward over the next five years the existing literature reports that the current trends identified above are here to stay and will intensify, and continue to reshape business models and 
the food buying and selling ecosystem, augmented by several broader trends:

- e-commerce: continued growth in online services, and q-commerce

- ecosystem transformation: growth in convenience, discount, and specialty stores; major grocers to move further into take-away and home-delivery market, while delivery aggregators and platforms will move further into retail. Online players will look to develop offline services

- data analytics/Al: will deliver rapid technological advances, and big data will grow ever more important to the consumer experience and competitive advantage

- food as a service: including recipe boxes and personalised nutrition; and a growing focus on personalised nutrition

- health and sustainability: organic, nutrition, provenance, local, food waste reduction, and environmental performance

Future trends are expected to emerge from within the current ecosystem through dynamic interactions and network effects between each of these trends creating new entrants, and more leverage for some players while driving other players out of business.

\section{Conclusion}

In summary, significant change is anticipated in the way we buy and sell food online over the coming years. As digital platforms and retailers increasingly move into new market areas, with overlapping roles, the responsibilities for food safety and consumer protection are becoming blurred. It is therefore recommended that FSA adopt a highly proactive anticipatory role in supporting industry to build food safety into its fabric from the start as novel business models and processes increasingly replace traditional ones. It is recommended to adopt a systems approach to regulation, integrating conceptual input from the field of network science to capture the realities of an increasingly dynamic, interactive, and networked food system.

\section{Food in the digital platform economy - making sense of a dynamic ecosystem reports}

\section{PDF}

View Executive summary: Food in the digital platform economy as PDF(Open in a new window) $(386.15 \mathrm{~KB})$

PDF

View Food in the digital platform economy - making sense of a dynamic ecosystem as PDF(Open in a new window) (1.97 MB) 